\title{
An Amateur CCD Camera
}

\author{
Christian Buil, Guylaine Prat, Eric Thouvenot \\ 28, rue Bonnat, F-31400 Toulouse, France
}

A CCD camera was built in 1984 using a linear detector (Thomson TH7810A) with 1728 pixels. The simplicity of this sort of detector enabled us to gain experience with CCDs. With a linear detector it is possible to carry out spectroscopy or even two-dimensional imaging by shifting the observed field at right angles to the photosensitive strip and by acquiring data in a regular manner (the scanning technique). The limitations of this equipment soon became apparent when trying to image deep-sky objects, so a second camera was built in 1985 using a CCD array, a Thomson TH7851 with $208 \times 144$ pixels. Numerous improvements were later made and the most advanced model is described.

\section{The CCD Camera}

Although the camera's principles are simple, it has very high performance, and technical solutions have made it very flexible in its applications, rapidly operational, and reliable. This has been largely because all the CCD's operations are controlled by a microcomputer, including charge transfer within the CCD. Windowing and binning, for example, are easily controlled by modification of instructions in a suitable program. Full computer-control has resulted in keeping the electronics to a minimum, with a consequent reduction in cost and ease of duplication. The computer is an IBM AT compatible, and the suite of programs is written in compiled BASIC. The greatest problems arise over the video output. Standard EGA graphics are used, allowing images of $640 \times 350$ pixels in 16 colours to be shown. VGA standards $(320 \times 200$ pixels but with 256 colours) are more pleasant to use.

The CCD is a Thomson TH7552A, $208 \times 144$ pixels, each 30 microns square, with raster-type charge transfer. The detector can be operated to prevent overloading, thus allowing observation of regions close to bright stars. This array costs about $2000 \mathrm{Fr}$ [approximately $\$ 340$ or $£ 200$ - Eds.]. Although larger arrays can be used, problems may occur with the amounts of data that have to be stored and manipulated (12-bit digitization of a $512 \times 512$ array would require $512 \mathrm{k}$ of memory).

Cooling is essential to minimize parasitic charges (dark current) - this detector will saturate in four seconds at $20^{\circ} \mathrm{C}$ - so the detector is cooled to $-50^{\circ} \mathrm{C}$ by 2 Peltier-effect thermoelectric stages, using a closed water-cooling circuit and a heat exchanger. The array is enclosed in a chamber held at about $10^{-3}$ Torr to avoid 
condensation on the CCD's optical window. Practical integration times range from a few tens of seconds to thirty minutes.

The camera's read-out noise is less than 100 electrons. This is obtained by two-stage numerical sampling (reference array - video array), a unique technique that has proved to be highly efficient and also allows the electronics to be further simplified.

The computer generates all the CCD control signals, and stores the 12-bit digitized signal. The conversion time for one pixel is 25 microseconds. A "Quick-Look" can be displayed on the EGA screen immediately after scanning. The acquisition program includes all the usual image-manipulation and presentation tools (various convolutions, arithmetical operations, isophotes, profiles, false colours...). This allows the quality of the images to be evaluated immediately after acquisition. An arithmetic co-processor is used (Intel 80287) and optimization of the compiled code has allowed high performance to be secured in implementation of two-dimensional Fast Fourier Transforms, for example.

Initially images are stored on a $20 \mathrm{M}$ hard disk and later backed-up onto $1.2 \mathrm{M}$ floppies. One image occupies approximately $60 \mathrm{k}$. Read and write times to the hard disk are about 1 second.

\section{Some Results}

The camera has been used on various telescopes, ranging from a small $150-\mathrm{mm}$ to the T 60 and the $1-\mathrm{m}$ at the Pic du Midi. The objects observed obviously varied with different telescopes, but on every occasion the CCD produced some surprises.

In planetary observation, the high sensitivity of CCDs allows short exposures, partly freezing atmospheric turbulence. The large dynamic range allows low-contrast details to be studied. This is one of the reasons we use 12-bit digitization of the video signal from the $C C D$, giving 4096 grey-levels. Such a range is unknown in photography. A standard CCD has a spectral range from 4000 to $10000 \AA$, and it is very instructive to observe the planets over such a range. Jupiter, for example, shows completely different appearances in the blue (high-contrast belts, few details) or in the infrared (low-contrast, but a mass of detail right up to the poles). The same planet observed with the $1-\mathrm{m}$ telescope through a filter with a narrow pass-band close to $8900 \AA$, corresponding to a methane absorption, shows a completely different aspect to the normal visual image: the poles are practically the brightest areas, while the temperate zones are very pale, and spots like the GRS appear that have no counterpart at other colours. We have also obtained a tri-colour image of Jupiter. The procedure is simple: one only has to acquire images of the planet at short timeintervals through three different filters (in our case: blue, green and infrared), and then numerically superimpose these images associating each with one of the primary colours. The result is a highly arresting colour image of Jupiter's cloud patterns. The radiometric quality of $\mathrm{CCD}$ images allows very faithful colours to be obtained after suitable calibration, and this would certainly not apply to ordinary colour film. We hope to obtain the first tri-colour images of galaxies in the near future. 
The most satisfying images were those obtained with a $150-\mathrm{mm}, \mathrm{f} / 5$ reflector, typical of instruments in amateur hands. The Ring Nebula (M57) is recognizable after only 5 seconds' integration. After 10 seconds the central star is visible; a 10minute integration shows the ring more or less saturated, but the very much weaker, outer ring is detectable. The small, 15.2-magnitude galaxy IC 1296, 4 arcminutes from M57 is easily visible. Comparing this image with the Palomar Sky Survey, stars of visual magnitude 20 are visible. Not bad for a $150-\mathrm{mm}$ telescope!

This must be qualified, however. The images were acquired without a filter, but for work of any astrophysical significance the CCD's range must be limited by BVRI-type filters (with pass-bands of about $1000 \AA$ ). With such a filter, and a 10 -minute exposure on the same instrument the limiting magnitude is about 17 in the red. A $400-\mathrm{mm}$ telescope ought to be able to reach magnitude 23 with a onehour exposure, which is practical with our present $\mathrm{CCD}$ cooling arrangement. At this level of detection it would be possible to study variable stars in M31 (which is well-resolved in just a 10 -minute exposure on the 150 -mm telescope).

A composite of two images, each of 10-minute integration, of the pair of galaxies NGC 5194 and 5195, better known as M51, clearly shows the material extending towards the west from NGC5195. When the image processing is "pushed", these extensions can be traced out to a surface magnitude of 25 per square arcsecond (according to the photometric study carried out by M.S. Burkhead: Astroph. J. Sup., $28,147-184,1978$ ). Such a magnitude is obtained because each pixel integrates energy arriving from a angle of more than 8 arcseconds on the sky. It should be noted that we obtained this image under considerable moonlight and quite a hazy sky. Under better conditions and with suitable correction, it would appear possible to reach a surface brightness of magnitude 27 . This result also shows that it is realistic to observe faint objects with considerable sky background.

\section{Conclusion}

As we have seen, the performance of CCDs is high and very appealing, out-classing photography in most applications, except for wide fields. Few amateurs are able to exploit photography to its limits. Work by David Malin, for example, has shown that image-amplification techniques allow considerable gains in detection to be achieved. But these gains are only acquired after a long series of careful trials and more hours spent in the darkroom than out observing. CCD images, on the other hand, all give optimum results. One importance aspect is that a CCD image is easier to exploit than a photograph, which too often is simply regarded as a work of art. A CCD image will give more information and will result in greater satisfaction at the end of the day. In the not-too-distant future it is even possible that an "image bank" will be set up, that one can consult by obtaining diskettes. Amateurs will probably have common methods of image processing, which will facilitate comparison of results.

It is clear that the introduction of the CCD is as important an event for astronomical observation as the introduction of photography was at the end of the last century. 